\title{
1 Emission of energetic neutral atoms from water ice under \\ 2 Ganymede surface-like conditions \\ 3
}

4 Martin Wieser $^{1^{*}}$, Yoshifumi Futaana ${ }^{1}$, Stas Barabash $^{1}$, Peter Wurz $^{2}$

5

$6 \quad{ }^{1}$ Swedish Institute of Space Physics, Box 812, SE-98128 Kiruna, Sweden

$7 \quad *$ email: wieser@irf.se

$8{ }^{2}$ Physikalisches Institut, University of Bern, Sidlerstrasse 5, CH-3012 Bern,

9 Switzerland

11 Abstract

12 The co-rotating plasma around Jupiter precipitates on the surfaces of the Jovian

13 moons, where it is not hindered by a local magnetic field. Precipitating ions lead to

14 the emission of energetic neutral atoms, which are produced via backscattering and

15 sputtering processes, from the surface. The European Space Agency's JUICE mission

16 to Jupiter carries as part of the Particle Environment Package experiment an imaging

17 energetic neutral atom spectrometer called the Jovian Neutrals Analyzer (JNA). When

18 it is in orbit around Ganymede, JNA will measure the energetic neutral atom flux

19 emitted from the surface of Ganymede in the energy range from $10 \mathrm{eV}$ to $3300 \mathrm{eV}$.

20 The surface of Ganymede consists of a large fraction of water ice. To characterize the

21 expected energetic neutral atom fluxes from water ice due to precipitating Jovian

22 plasma, we impacted protons and singly charged oxygen ions with energies up to

$2333 \mathrm{keV}$ on a salty water ice target kept at Ganymede surface conditions. Emitted

24 energetic atoms were measured energy- and mass-resolved using the JNA prototype

25 instrument. The data show high yields for energetic neutral atoms per incident ion in 
26 the JNA energy range. For incident protons, energetic neutral atom yields between

$27 \quad 0.28$ at $1 \mathrm{keV}$ and $\sim 40$ at $33 \mathrm{keV}$ were observed. For incident singly charged oxygen

28 ions, the observed energetic neutral atom yield ranged from 0.8 for at $3 \mathrm{keV}$ to $\sim 170$

29 at $23 \mathrm{keV}$.

30

31 Keywords:

32 Ganymede, Ice, Plasma-surface interaction, Energetic neutral atoms

34 Highlights:

35 Sputtering by ions from water ice under Ganymede surface conditions are

36 investigated

37 Mass resolved energy spectra of sputtered energetic neutral atoms are measured

38 High sputter yields of energetic neutral atoms by impinging ions are observed

\section{Introduction}

41 Jupiter possesses a large magnetosphere that extends far beyond the orbits of the

42 Galilean moons. These moons are constantly exposed to the co-rotating flow of

43 Jovian magnetospheric plasma (Khurana et al., 1997). In the absence of an

44 atmosphere and when it is not hindered by an intrinsic local magnetic field, this

45 plasma reaches the surface and interacts directly with surface materials. The

46 sputtering of surface materials by incident ions results in a flux of energetic neutral

47 atoms (ENA) leaving the surface (Shi et al. 1995; Ip et al., 1997; Johnson et al. 1998;

48 Cooper et al. 2001; Baragiola 2003, Famá et al. 2008) that contribute to the

49 formation of extended exospheres. The energy distribution of these ENAs is 
dominated by energies below 10s of eV (Siegmund, 1969; Betz and Wien, 1994) and, in the context of planetary science, has a cutoff at approximately $100 \mathrm{eV}$ (Wurz and Lammer, 2003; Wurz et al., 2007). A fraction of the impinging ion flux is expected to backscatter to space as ENAs, despite the large expected surface roughness. For comparison, from the regolith covered rocky surface of the Moon, up to $20 \%$ of the impinging solar wind protons are backscattered as hydrogen ENAs (Wieser et al. 2009). These backscattered ENAs have substantial energies far above those of the ENAs produced by electronic sputtering, with up to $50 \%$ of the impinging solar wind protons (Wieser et al. 2009, Futaana et al. 2012). It is expected that in the Jovian system, a similarly large fraction of the impinging co-rotating plasma ions are backscattered from the icy moons' surfaces as ENAs. On Ganymede, the surface is partially shielded from the magnetospheric plasma inflow by Ganymede's intrinsic magnetic field (Cooper et al., 2001, Khurana et al 2007). Imaging the ENA flux from the surface will make visible the boundaries of the regions, where plasma is able to

64 reach the surface nevertheless, i.e., at the polar caps. This method is similar to how magnetic anomalies on the Moon were imaged (Wieser et al., 2010). Experimental data for the emission of ENAs from ices as found, e.g., on Ganymede's surface (Showman and Malhotra, 1999) when it is bombarded with ions in the keV to 10s of keV energy range, usually focus on the total sputter yield; thus, little data exist

69 regarding the energy spectra of the emitted ENAs (Shi et al. 1995; Ip et al, 1997;

70 Johnson et al. 1998; Baragiola 2003; Famá et al. 2008). This is due to the difficulty 71 in measuring ENAs in the energy range from 10 s of eV up to a few $\mathrm{keV}$.

72 We present mass resolved measurements of the emitted ENA energy spectrum from $7310 \mathrm{~s}$ of $\mathrm{eV}$ up to a few $\mathrm{keV}$ that were obtained from an icy surface bombarded with 
74 positive ions. We estimate energetic neutral atom yields for emitted ENAs with energies above $15 \mathrm{eV}$. The experiment was performed in the MEFISTO test facility at the University of Bern (Marti et al., 2001). A prototype model of the Jovian Neutrals Analyzer (JNA) was used as the energy and mass resolving ENA detector. The JNA instrument is based on the Energetic Neutral Atom instrument (Kazama et al., 2006), which is part of the payload of the Mercury Magnetospheric Orbiter of the BepiColombo mission to Mercury. A similar instrument, the Chandrayaan Energetic Neutral Atom analyzer, was successfully flown on the Indian Chandrayaan-1 mission to the Moon (Barabash et al. 2009). JNA was selected to fly as part of the Particle Environment Package (PEP) (Barabash et al. 2013) on ESA's Jupiter Icy moons Explorer (JUICE) (Grasset et al. 2013). The science objectives of JNA are closely tied to the interaction of ions with ices. While it is in orbit around Ganymede, JNA will map the precipitation pattern of ions on the surface via sputtered and backscattered ENAs. JNA measurements will constrain the magnetic field topology on the surface of Ganymede, analogous to CENA measurements on the Moon (Wieser et al. 2010). JNA will also observe the dynamics of the Io plasma torus remotely via ENAs that are generated through charge exchange with the neutral gas background (Futaana et al. 2015).

\section{Experimental setup}

The experimental setup (Figure 1) consisted of an ice disk exposed to an incident ion beam. Energetic neutral atoms emitted from the ice disk were measured using a prototype of the JNA instrument. The JNA prototype was mounted such that it measured energetic neutral atoms emitted at angles centered on the specular reflection 
98 direction from the ice disk. The pencil ion beam provided by the MEFISTO test

99 facility was $5 \mathrm{~mm}$ in diameter and directed at a grazing incidence angle $\Phi$ of $83^{\circ}$ to

100 the surface normal to the ice disk (Figure 1). Monoenergetic beams of $\mathrm{H}^{+}$and $\mathrm{O}^{+}$ions

101 were used, with energies between $1 \mathrm{keV}$ and $33 \mathrm{keV}$. The ion beam intensity was

102 periodically measured with a Faraday cup. Typical ion beam currents were on the 103 order of $1 \mathrm{nA}$.

104

105

106

107 Figure 1: Experiment geometry: An ion beam from the left hits the ice disk at a 108 grazing incidence of $\Phi=83^{\circ}$ to the surface normal. The JNA prototype instrument 109 records energetic neutral atoms emitted around the specular reflection direction $\theta=$ $11083^{\circ}$

111

112 The ice disk was prepared from a mixture of $11 \mathrm{~g} \mathrm{NaCl}$ and $150 \mathrm{ml} \mathrm{H}_{2} \mathrm{O}$, frozen in a

113 plastic dish placed on dry ice. The salt concentration corresponded to approximately

114 twice the salt concentration found in typical terrestrial seawater. Ice from such a

115 solution contains two distinct phases: $\mathrm{H}_{2} \mathrm{O}$ (water ice) and $\mathrm{NaCl} \cdot 2 \mathrm{H}_{2} \mathrm{O}$ (hydrohalite),

116 with the water ice phase dominating (e.g., McCarthy et al., 2007). $\mathrm{NaCl}$ was added to

117 simulate impurities in the ice block and to provide some electrical conductivity. The

118 latter prevented the buildup of charges on the scattering surface during ion

119 bombardment. The ice block was then roughly shaped using very coarsely grained

120 glass sand paper to a flat disk of approximately $10 \mathrm{~cm}$ in diameter and a few $\mathrm{mm}$ in 
121 thickness. The surface roughness $R_{a}$ after polishing was estimated to be $0.2 \mathrm{~mm}$. The

122 resulting ice disk was white in color due to small-scale ice grain boundaries and

123 additional frozen $\mathrm{CO}_{2}$ bubbles originating from the dry ice. Figure 2 shows the ice

124 disk installed in the vacuum system. Vacuum conditions near the ice surface were

125 determined by the vapor pressure of the ice. The conditions on the surface of

126 Ganymede correspond well to a point on the vapor pressure curve for water at $10^{-7}$

127 mbar and a temperature of $150 \mathrm{~K}\left(-123{ }^{\circ} \mathrm{C}\right)$. (Murphy et al., 2006). A dedicated liquid

128 nitrogen cooling system, keeping the temperature of the ice within a few degrees of

$129150 \mathrm{~K}$, supported the ice disk. This temperature and the resulting vacuum pressure of

130 approximately $10^{-7}$ mbar were maintained throughout the scattering experiment. At

$131150 \mathrm{~K}$, the $\mathrm{CO}_{2}$ enclosed in bubbles in the ice should freeze out. It is unlikely,

132 however, that pure $\mathrm{CO}_{2}$ ice was exposed to the ion beam. The vapor pressure of pure

$133 \mathrm{CO}_{2}$ ice at $150 \mathrm{~K}$ is approximately 13 mbar (James et al., 1992), and exposed $\mathrm{CO}_{2}$ ice

134 would have already sublimated quickly during the pump down process of the vacuum

135 system. Indeed, $\mathrm{CO}_{2}$ pressure spikes were observed during the early phases of the

136 vacuum pump down process. However, a small amount of $\mathrm{CO}_{2}$ was likely present in

137 the ice as a mixed phase ice. $\mathrm{CO}_{2}$ bound in surface ices was observed on Ganymede

138 using infrared spectroscopy (McCord et al. 1997, 1998). However, we estimate that

139 the exposed part of our ice disk contained less than $1 \mathrm{CO}_{2}$ molecule per $100 \mathrm{H}_{2} \mathrm{O}$

140 molecules, based on the monitored residual gas composition. A similar small amount

141 of air may have been included in bubbles in the ice.

142 The JNA prototype measures energetic neutral atoms entering its aperture by

143 reflecting them at grazing incidence on a charge conversion surface and thereby

144 positively ionizing them. The newly generated positive ions are then energy analyzed 
145 and post-accelerated to keV energy and are finally detected in a time-of-flight section.

146 Ions are prevented from entering the aperture by a charged particle deflection system.

147 A detailed description of the ion optics can be found in Kazama et al. (2006). For this

148 experiment, the JNA energy analyzer was programmed with 8 logarithmically spaced

149 nominal center energies $(20 \mathrm{eV}, 40 \mathrm{eV}, 80 \mathrm{eV}, 160 \mathrm{eV}, 320 \mathrm{eV}, 640 \mathrm{eV}, 1280 \mathrm{eV}$ and

$1502560 \mathrm{eV})$. Combined with an energy resolution $\Delta \mathrm{E} / \mathrm{E}$ of approximately $100 \%$, this

151 resulted in dense coverage of an energy range from $15 \mathrm{eV}$ to $3300 \mathrm{eV}$. Actual center

152 energies are slightly species dependent due to the species dependent energy loss at the

153 charge conversion surface of JNA. The angular resolution of the JNA prototype was

$15425^{\circ}$ times $7^{\circ}$ full width at half maximum; however, given the experimental setup used,

155 the ENA emitting spot on the ice sample, as seen from JNA, was always much smaller

156 than this and was completely contained within one angular pixel. The mass resolution

157 of JNA allowed the separation of mass groups centered on m=1 amu ("H"), 4 amu, 16

158 amu ("O-group") and >32 amu ("heavy"). The O-group may also contain a $\mathrm{H}_{2} \mathrm{O}$

159 component that cannot be distinguished from atomic oxygen due to a mass difference

160 that is too small. In this paper, we generally use the term 'oxygen group ENAs' when

161 referring to this oxygen/water mass group.

162 Calibration of ENA sensors at energies below $1 \mathrm{keV}$ is a challenge, and the

163 JNA instrument is no exception. For the measurements shown, we combined the

164 instrument performance data obtained from flight units of the ENA sensors flown on

165 Chandrayaan-1 and on BepiColombo. Both instruments are representative with

166 respect to the performance of the JNA prototype used in this experiment. Energetic

167 neutral beams of hydrogen and oxygen, produced by the MEFISTO system ion beam

168 neutralizer (Wieser and Wurz, 2005), were used to confirm the sensitivity of the JNA 
prototype. The MEFISTO system is able to produce an energetic neutral hydrogen or

170 oxygen beam in the energy range from $10 \mathrm{eV}$ to $3 \mathrm{keV}$, with an energy width of

171 approximately $15 \%$ for neutral hydrogen and approximately twice that value for

172 neutral oxygen. This energy width is still small compared with the energy resolution

173 of the JNA sensor.

174

175

176

177

178

179

180

181

182

183

184

185

186

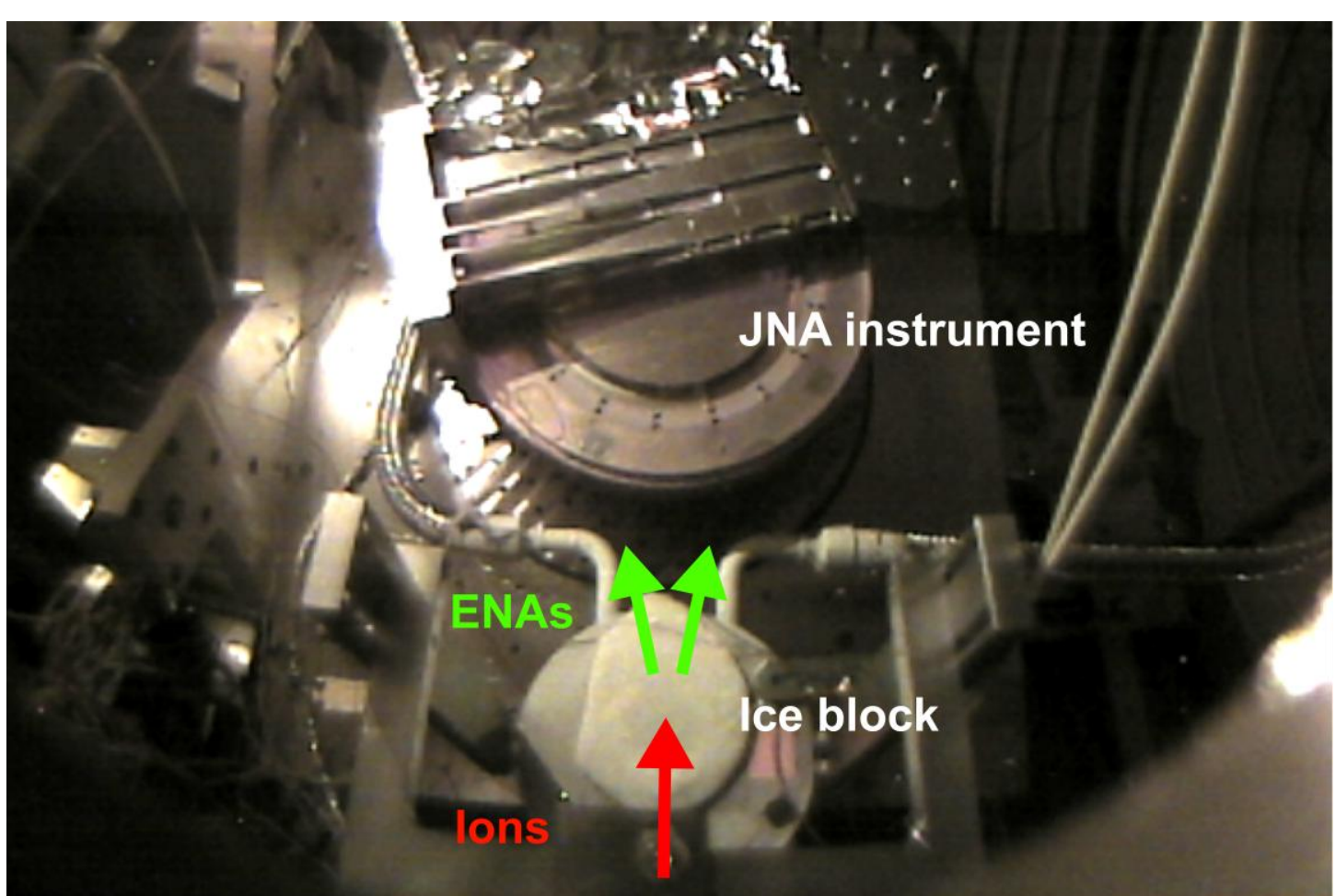

Figure 2: View into vacuum system during the measurements, with ice block (bottom center) and JNA prototype instrument (top center). The ion beam hits the ice block from the lower edge of the image (red arrow) and ENAs are scattered towards the JNA instrument (green arrows). Below the ice disk, the cooled support with liquid nitrogen feeding lines is visible.

\section{Results}

A total of 6 different ENA production channels were investigated: $\mathrm{H}^{+}$and $\mathrm{O}^{+}$ incident ions producing $H, O$ and heavy ENAs, with heavy indicating ENAs with a mass significantly larger than that of atomic oxygen and a resulting time-of-flight much longer than that of oxygen. Whereas mainly $H$ - and $O$-group ENAs are released 
187 from the surface for incident protons up to $33 \mathrm{keV}$, the heavier $\mathrm{O}^{+}$projectiles also 188 efficiently sputter $\mathrm{Na}$ and $\mathrm{Cl}$ atoms, water molecules, $\mathrm{O}_{2}, \mathrm{H}_{2} \mathrm{O}_{2}$ or complexes of 189 several water molecules from the ice block with significant efficiency. All of these are 190 candidates for the observed heavy ENA component with very long time-of-flight, but 191 not all of them are equally well detected by JNA: water complexes are very likely 192 sputtered from the ice (Lancaster et al., 1979) but have a rather low probability to 193 remain intact during the interaction with the charge conversion surface in the JNA 194 prototype sensor. $\mathrm{H}_{2} \mathrm{O}_{2}$ produced by ion irradiation of the target (Loeffler et al., 2006) 195 is also a candidate for the heavy ENA component, but the expected concentrations are 196 small, and again, it is unlikely that this molecule survives the charge conversion 197 surface interaction in the JNA sensor intact. The JNA detection efficiency for chlorine 198 is low due to its large electro-negativity; it would need to form a positive chlorine ion 199 during interaction with the JNA charge conversion surface. It is thus unlikely that the 200 heavy signal is due to the detection of chlorine. The most likely candidate for the 201 measured signal is Na. Sodium is likely ionized on the instrument's charge conversion 202 surface due to its low ionization energy, and it should thus be easily detected by the 203 JNA prototype. Unfortunately, the detection efficiency of the JNA prototype for 204 energetic neutral $\mathrm{Na}$ or $\mathrm{Cl}$ or possible water clusters and the shape of the 205 corresponding time-of-flight spectra are not known at present; they will be determined 206 during JNA flight model calibrations. For the analysis shown here, it was assumed 207 that the water molecules and the heavy component have the same detection efficiency 208 as energetic oxygen, and the shape of the time-of-flight spectrum of the heavy component was empirically determined.

210 Signal strength in each of the ENA mass groups was determined by fitting $\mathrm{H}$, 
211 O-group and residual "heavy" components to the measured time-of-flight spectra for

212 each energy band separately. The resulting count rates for each mass group were then

213 converted using the JNA geometric factor to differential number flux $j$ in units $\left(\mathrm{cm}^{-2}\right.$

$\left.214 \mathrm{sr}^{-1} \mathrm{eV}^{-1} \mathrm{~s}^{-1}\right)$. To compensate for a varying incident ion beam intensity, this differential

215 flux was then normalized using the incident ion beam flux, which resulted in a 216 normalized ENA flux $F$ in units $\left(\mathrm{sr}^{-1} \mathrm{eV}^{-1}\right)$ :

$$
F(E, p, s)=\frac{j(E, s)}{I(p)},
$$

218 where $I$ is the integrated flux of the impinging ion beam in units $\left(\mathrm{cm}^{-2} \mathrm{~s}^{-1}\right), p$ is the

219 incident ion species and $s$ is the measured ENA species. The value of $F$ depends on 220 the experiment geometry; the values presented here are specific for the geometry

221 shown in Figure 1. Figure 3 shows a summary of the measured energy spectra of the

222 normalized flux for different combinations of primary beam and secondary particle

223 species. For the data shown, the error bar includes the uncertainty of the geometric

224 factor and the uncertainty due to counting statistics. As a general trend, the observed

225 ENA intensities are higher for a larger ratio of projectile mass to emitted ENA mass,

226 e.g., protons are rather inefficient for producing heavy ENAs (Figure 3e), whereas

227 incident oxygen ions produce more than an order of magnitude larger ENA fluxes

228 (Figure 3f). 

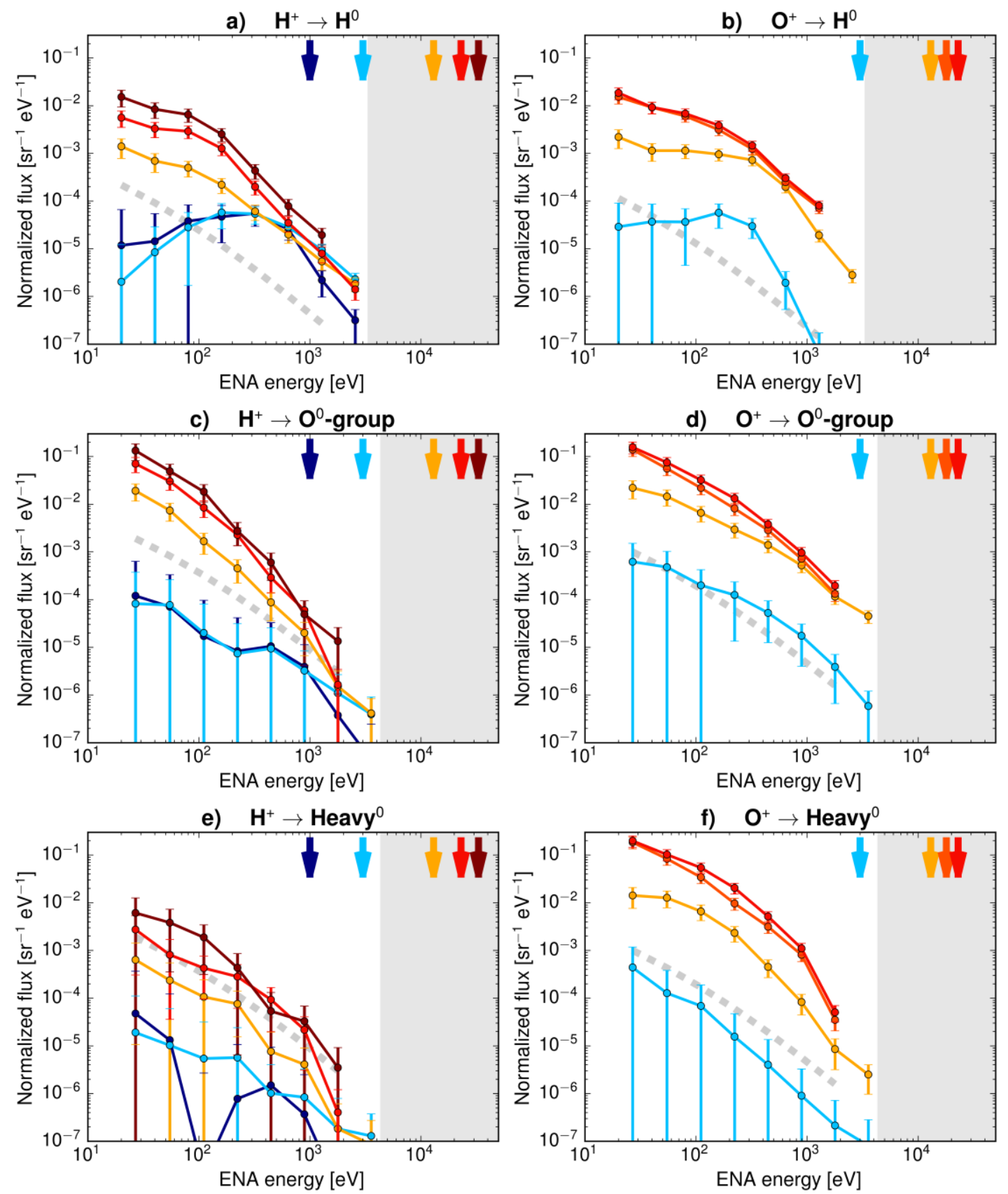

Figure 3: ENAs spectra measured for incident protons (panels $a, c$ and $e$ ) and incident oxygen ions (panels $b, d$ and $f$ ) and different emitted ENA mass groups $(\mathrm{H}, \mathrm{O}$ group and, Heavy). The energy spectra are normalized with the incident flux onto the ice target. The colored arrows represent the incident ion beam energy, which belongs to an energy spectrum of the same color. The gray dashed line represents an average one-count limit for the measurements shown. The ENA energy range not accessible by the JNA sensor is denoted with a gray background. 


\section{Discussion}

\section{$243 \quad 4.1$ Energy spectra}

244 The recorded energy spectra can be divided into four types (or a combination of 245 these): (1) spectra where the incoming particles are directly reflected as ENAs 246 (Figures 3a and 3d), (2) spectra where recoil particles are generated from the ice 247 surface (collisional sputtering, Figure 3b), (3) energy spectra where the incident species has too high energy to be directly recorded as reflected ENAs and where products from electronic sputtering dominate in the energy range where measurements are made (Figure 3, all panels for ion energies $>3 \mathrm{keV}$ ), and (4) $\mathrm{H}$ ENA spectra obtained from incident oxygen ions with sufficiently high energy. In this case, the incident ion is heavy enough to produce a significant molecular $\mathrm{H}_{2} \mathrm{O}$ ENA flux that likely dissociates at the instruments charge conversion surface, thus creating an artificial H ENA signal (Figure 3b, for incident ion energies of $18 \mathrm{keV}$ and above). Examples of these four types are discussed in detail below.

Figure 4 shows an example of the first type of energy spectrum of reflected hydrogen ENAs generated from incident low energy $(1 \mathrm{keV})$ protons, along with a

258 fitted Maxwell distribution, as derived by Futaana et al. (2012). The observed energy spectrum is well reproduced by the fit, which suggests that the particles are scattered

260 from the ice surface similar to particles scattered from the regolith on the lunar 261 surface. For incident $1 \mathrm{keV}$ protons, the characteristic energy of hydrogen 262 backscattered from the lunar regolith is $k_{B} T=117 \mathrm{eV}$ (Futaana et al., 2012). For the 263 ice scattering case $k_{B} T=165 \mathrm{eV}$ was found. This value is somewhat sensitive to the 264 shape of the JNA instrument energy acceptance function. Variations of this function within its confidence limits resulted in characteristic energies ranging from $146 \mathrm{eV}$ to 
$191 \mathrm{eV}$ for the same data. These characteristic energies are 1.5-2 times higher than

267 the typical case of scattering from the lunar regolith. This may be due to being much

268 closer to the normal angle of incidence in the lunar regolith case shown in Futaana et 269 al. (2012) compared with the grazing angle of $83^{\circ}$ used in this study. The obtained 270 reflection fraction from ice of 0.28 for incident protons at $1 \mathrm{keV}$ is, however, almost the same as the hydrogen reflection coefficient of 0.19 reported for the lunar regolith, as measured using an almost identically built instrument on Chandrayaan-1 (Wieser et al., 2009; Futaana et al., 2012; Vorburger et al., 2013).

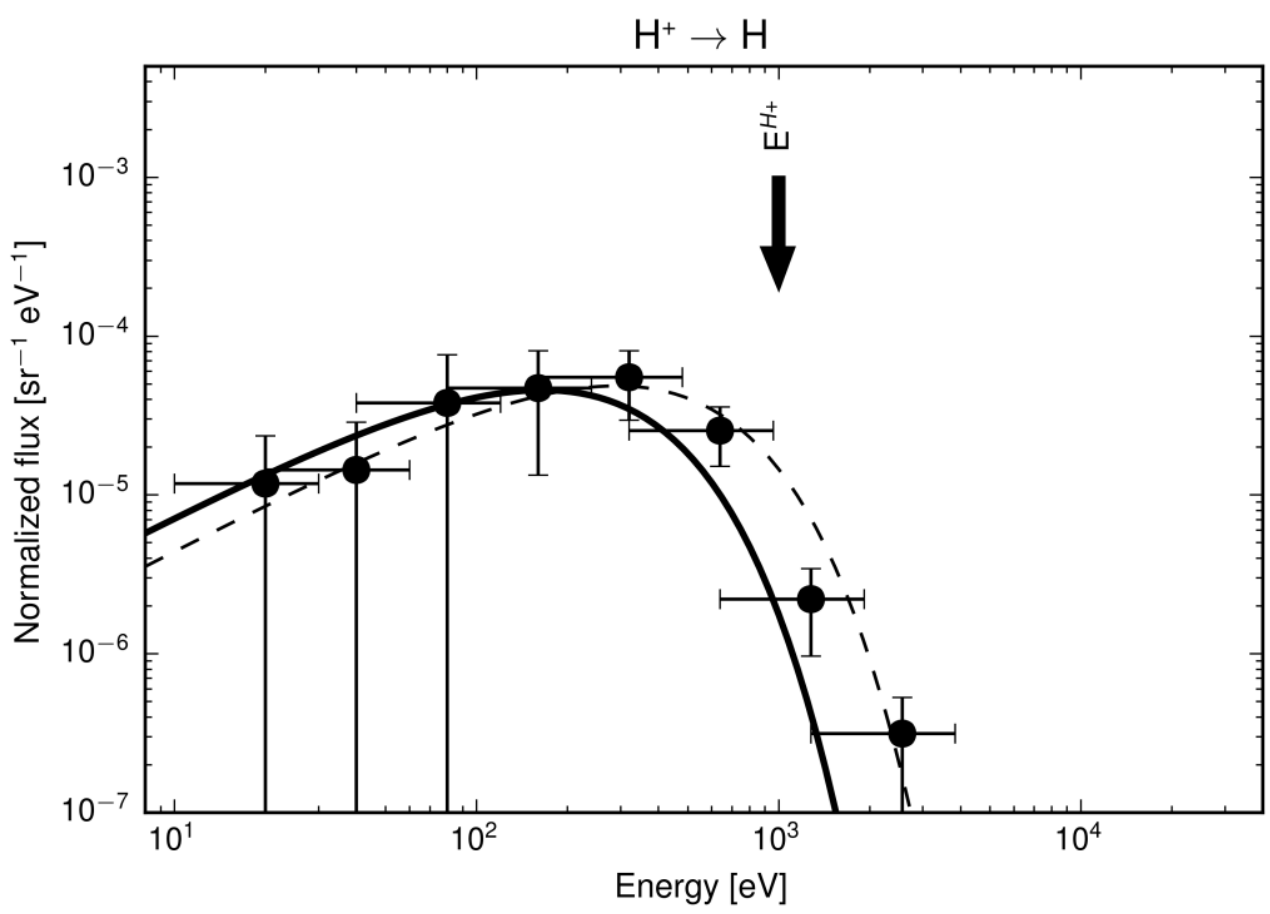

275

Figure 4: Measured energy spectra of the hydrogen ENA differential flux from the ice surface, normalized to the incident flux prior to deconvolution of the instrument energy response function (circles) for an incident $1 \mathrm{keV}$ proton beam (arrow). A fit to the measured energy spectra consisting of a Maxwell distribution convoluted with the instrument energy response is shown as a dashed line. The Maxwell component only is shown as a solid line and has a characteristic energy of $k_{B} T=165 \mathrm{eV}$. Error bars on the $x$-axis indicate the energy bin width of the instrument; error bars on the y-axis represent measurement uncertainties. 
An example of the second type of energy spectrum is shown in Figure 5:

287 incident oxygen ions with $3 \mathrm{keV}$ energy create recoil hydrogen ENAs. Their upper

288 energy limit is well modeled by a single elastic collision of the $\mathrm{O}^{+}$projectile with a

289 hydrogen atom on the surface (Niehus et al., 1993). In general, collisional sputtering

290 dominates at low energies, with total yields that are fairly independent of incident

291 energy (Johnson, 1998). The energy $\mathrm{E}_{\mathrm{R}}$ of a target recoil particle from a single elastic

292 collision is given by

$293 E_{R}=E \frac{4 A \cos ^{2}}{(1+A)^{2}}$, for $90^{\circ}$

294 with $M_{1}$ being the mass of the projectile, $M_{2}$ being the mass of the recoil particle and

295 the mass ratio $A$ defined as $A=M_{2} / M_{1}$. E denotes the incident ion energy and $\delta=180^{\circ}$ -

$296 \theta-\phi$ is the total deflection angle for this experiment (Figure 1).

297

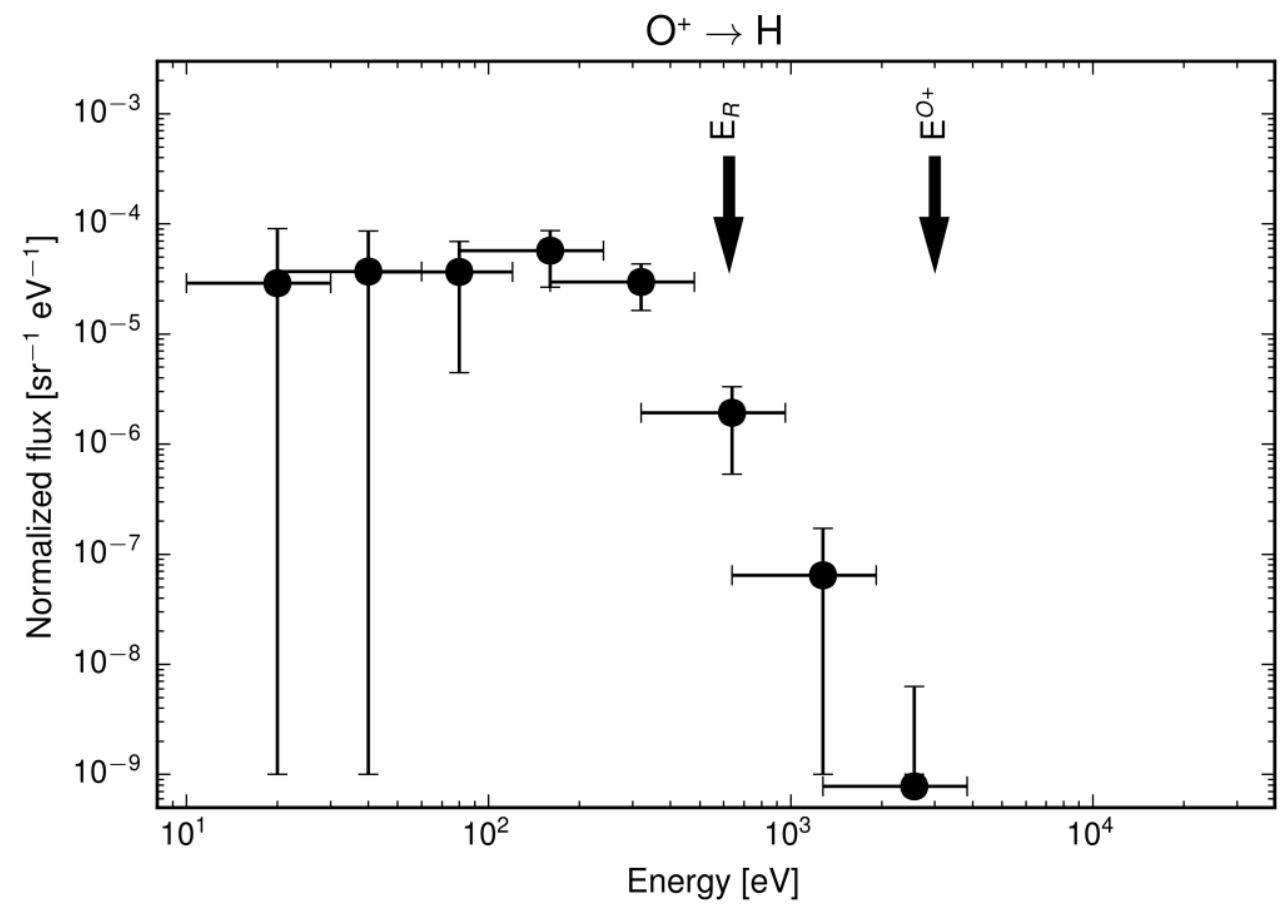


299 Figure 5: The energy spectrum of the hydrogen ENA differential flux from the ice 300 (circles) for incident $O+$ ions with $E^{O+}=3 \mathrm{keV}$ (right arrow). An upper energy limit 301 for the recoil energy is at $E_{R}=625 \mathrm{eV}$ (left arrow). Error bars have the same meaning 302 as in Figure 4.

303

An example of the third type of energy spectra is shown in Figure 6, i.e., the energy spectrum of neutral oxygen produced from an incident $33 \mathrm{keV}$ hydrogen ion

306 beam. Fitting with a Maxwell distribution does not reproduce the shape of the 307 measured spectrum. This indicates that the observed ENAs are not due to a single 308 collision scattering process or collisional sputtering. A reasonable fit for the energy 309 spectrum $F(E)$ is obtained using a Thompson-Sigmund formula that models electronic 310 sputtering (Sigmund, 1969), modified to account for the masses of the projectile and 311 the emitted ENA (Betz and Wien, 1994):

$$
\begin{aligned}
& F(E) \mu \frac{E}{\left(E+E_{b}\right)^{3}} 1 \sqrt{\frac{E}{E_{b}+E_{i}^{\prime}}} \div \\
& E_{i}^{\prime}=4 E_{i} \frac{M_{1} M_{2}}{\left(M_{1}+M_{2}\right)^{2}}
\end{aligned}
$$

313 where $\mathrm{E}$ is the energy of the emitted ENA, $\mathrm{E}_{\mathrm{b}}$ is the surface binding energy of $2-4 \mathrm{eV}$

314 (Wurz and Lammer, 2003), and $\mathrm{M}_{1}$ and $\mathrm{M}_{2}$ are the mass of the projectile and the 315 emitted ENA, respectively. The spectrum has a characteristic cut-off energy of $\mathrm{E}_{\mathrm{co}}=\mathrm{E}_{\mathrm{i}}{ }^{\prime}$

$316-\mathrm{E}_{\mathrm{b}}$, which, for this example, is above the energy range covered by the experiment. 


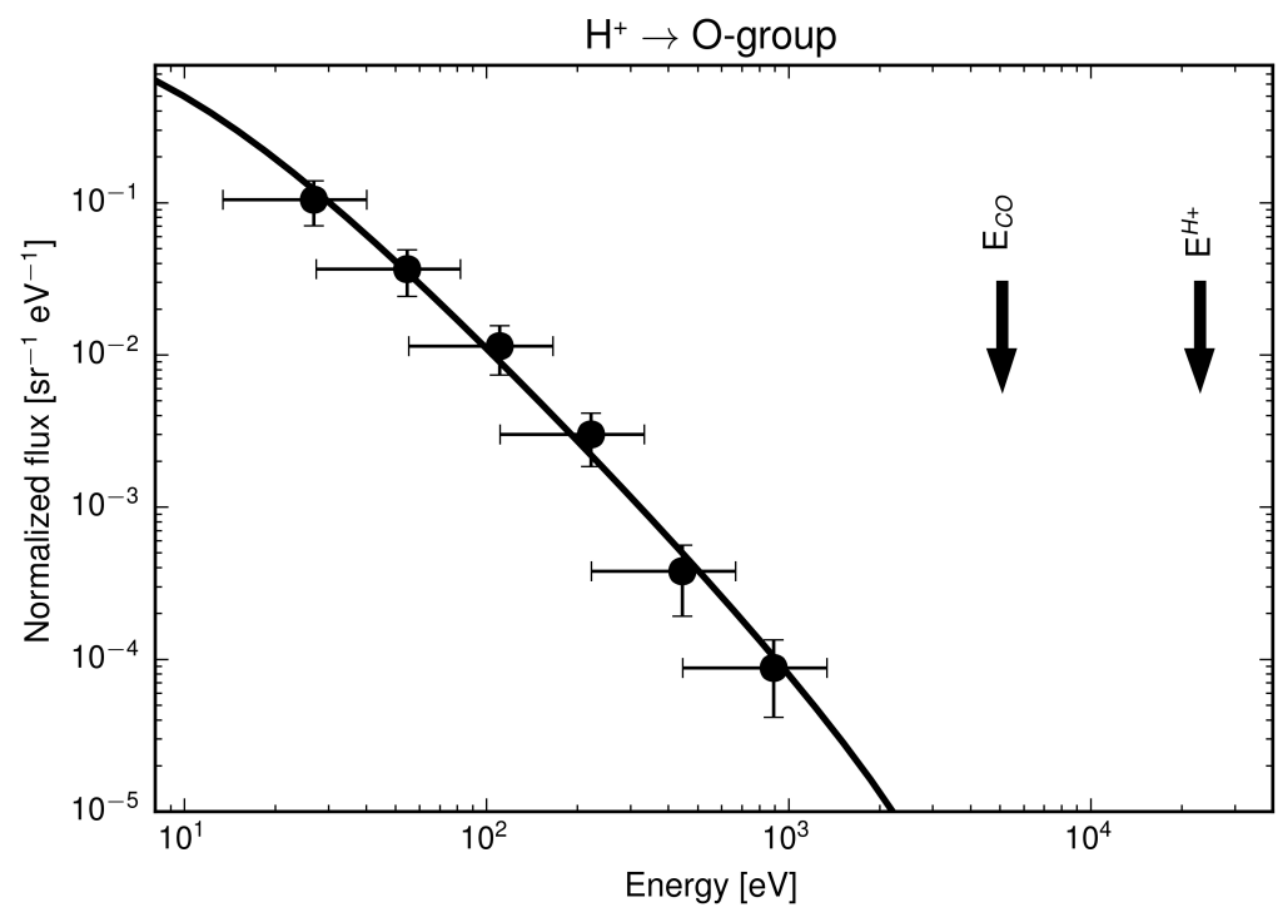

317

318

319

320

321

322

325

326

327

328

330

331

332

333

Figure 6: Energy spectrum of oxygen group ENAs sputtered by the $33 \mathrm{keV}$ proton beam (circles). The right arrow indicates the energy of the impinging ions. A fit using the Thompson-Sigmund spectra (solid line) and its cutoff energy $E_{C O}$ of $5100 \mathrm{eV}$ (left arrow) are also shown. Error bars have the same meaning as in Figure 5.

A fourth type of observed energy spectra is related to H ENA spectra only; Figure 7 shows such a H ENA spectrum, along with the corresponding O-group ENA spectrum, both of which are produced from the same incident $23 \mathrm{keV}$ oxygen ion beam. The H ENA component is well modeled by assuming that the O-group signal consists entirely of energetic $\mathrm{H}_{2} \mathrm{O}$ molecules and that some of these molecules dissociate at the instruments charge conversion surface, thus creating an artificial $\mathrm{H}$ ENA signal. Energy dependent dissociation fractions for incident $\mathrm{H}_{2} \mathrm{O}$ ENAs at the JNA charge conversion surface are, unfortunately, not yet known. A simple model for this artificial H ENA signal reproduces at least the low-energy part of the H ENA spectrum. In this model, complete dissociation for $1280 \mathrm{eV} \mathrm{H}_{2} \mathrm{O}$ molecules at the charge conversion surface and an energy proportional dissociation fraction (van Slooten et al, 1991) below $1280 \mathrm{eV}$ are assumed. Because the dissociation occurs 
336 prior to energy analysis, the hydrogen fragments appear downshifted in the energy 337 spectrum, with an energy $\mathrm{E}_{\mathrm{H}}=\mathrm{E}_{\mathrm{H} 2 \mathrm{O}} / \mathrm{m}_{\mathrm{H} 2 \mathrm{O}} * \mathrm{~m}_{\mathrm{H}}$ if no energy losses on the charge 338 conversion surface are considered (Figure 7, H-model). This model can explain all of 339 the H ENA flux below $100 \mathrm{eV}$. However, the same model applied to lower energy 340 incident oxygen ions $(\mathrm{E}<18 \mathrm{keV})$ predicts more than an order of magnitude larger $\mathrm{H}$ 341 ENA fluxes than were observed. Thus, in these cases, the O-group ENAs likely consist mostly of atomic oxygen and not water molecules.

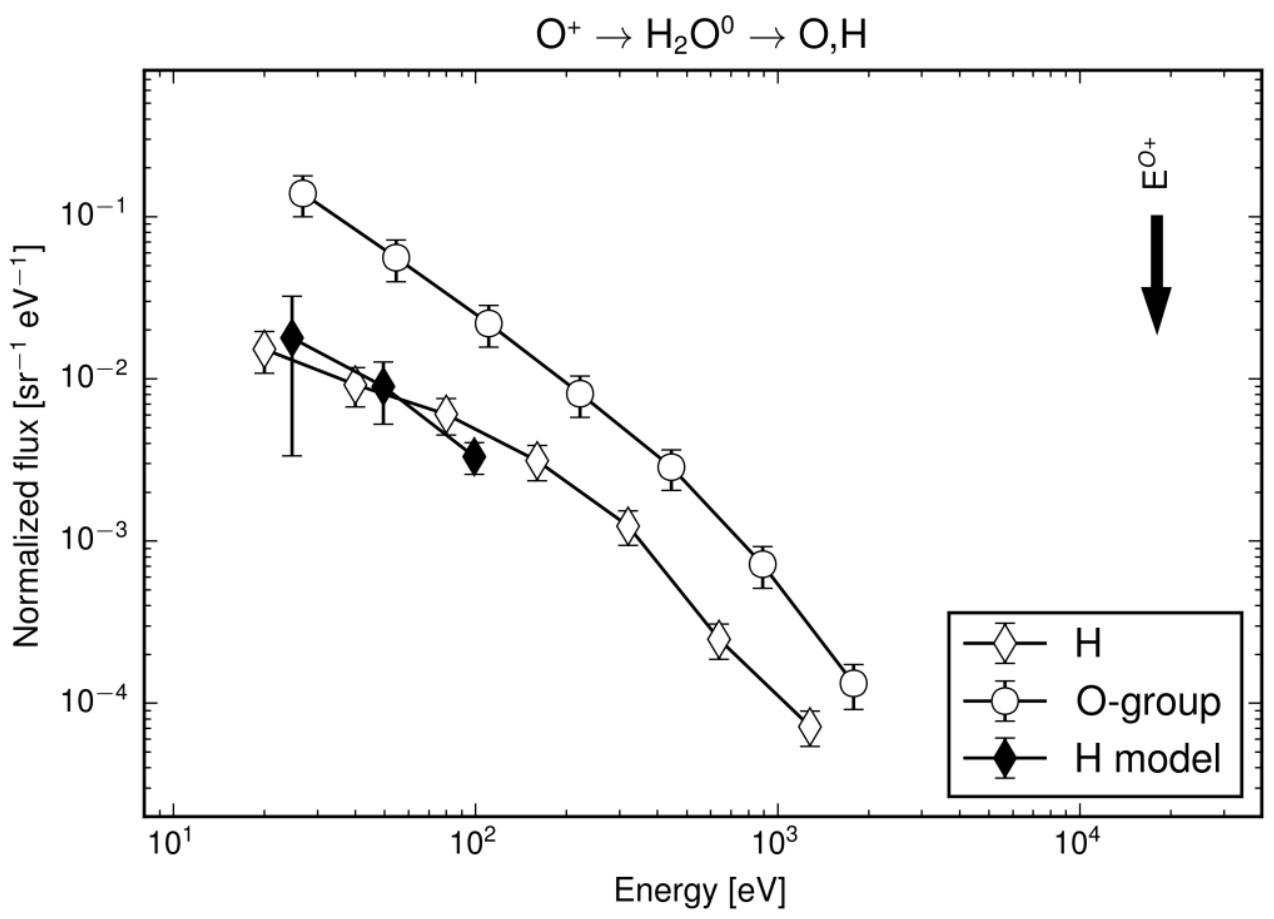

343

$344 \quad$ Figure 7: Energy spectrum of oxygen-group ENAs sputtered by the $23 \mathrm{keV}$ oxygen ion 345 beam (open circles) and the corresponding $H$ ENA signal (open diamonds). The $H$ 346 ENA signal is compatible with all of the oxygen-group ENAs and is $\mathrm{H}_{2} \mathrm{O}$ dissociative 347 at the JNA charge conversion surface, at least for energies above 200eV. The modeled $348 \mathrm{H}$ ENA background, originating from the dissociation of $\mathrm{H}_{2} \mathrm{O}$ ENAs, is shown with 349 filled diamonds.

\subsection{ENA yield}

From Figure 3, the energetic neutral particle yield $\mathrm{Y}_{\mathrm{ENA}}$, which is the ratio

353 between the total number of emitted energetic neutral particles above an energy 
354 threshold $\mathrm{E}_{\min }$ and the total number of incident ions, is calculated as the angular 355 integral of the normalized ENA flux, $F$. Assuming a non-isotropic angular distribution 356 of the emitted particles proportional to $\cos ^{4 / 3} \theta$, where $\theta$ is the angle to the surface 357 normal (Vidal et al. 2005), the integral ENA yields $\mathrm{Y}_{\mathrm{m}}$ for the three mass groups $\mathrm{m}=H, O$ or Heavy in the energy range covered by JNA are estimated by using

$$
\begin{aligned}
& Y_{m}=k_{E_{\min }}^{E_{\max }} F_{m}(E) d E \\
& Y_{E N A}=Y_{H}+Y_{O}+Y_{\text {Heavy }}
\end{aligned}
$$

360 with $\mathrm{E}_{\min }=15 \mathrm{eV}$ and $\mathrm{E}_{\max }=3.3 \mathrm{keV}$. The factor $\mathrm{k}$ ensures proper normalization to 361 the assumed $\cos ^{4 / 3} \theta$ angular distribution of the emitted ENA flux and the actual 362 observation direction of $83^{\circ}$ to the surface normal. A summary of yields is shown in 363 Figure 8 . When adding the yields for the different mass groups, the total energetic 364 neutral particle yield $\mathrm{Y}_{\mathrm{ENA}}$ is obtained. An empirical model of sputtering yields from 365 pure water ice surfaces is described by Famà et al. (2008) and shown for comparison. 366 Data for this model were mostly derived from relatively flat thin ice films (e.g., 367 Baragiola et al., 2003). Sputter yields for rough surfaces are similar to the sputter 368 yields of flat surfaces at smaller incidence angles (Küstner et al., 1998). This is 369 because for a rough surface and a given fixed macroscopic incidence angle, on the 370 microscopic level, which is seen by the impinging particles, a broad range of 371 incidence angles occur at the same time. Furthermore, on a rough surface, the sputtering yield is lower than that of a flat surface due to the re-deposition of sputtered products (Küstner et al., 1999; Loeffler et al., 2009). We estimate that the

374 sputtering yields at an $83^{\circ}$ macroscopic angle of incidence, as used in our setup, 375 correspond approximately to the sputtering yields from a flat surface at an $\sim 60^{\circ}$ angle 376 of incidence (Küstner et al., 1999). Including this correction for the incident angle, 
our measurements are compatible with the model of Famà et al. (2008), as discussed

378 below, despite the ice temperature of $150 \mathrm{~K}$ being outside the parameter range this model was made for $(\mathrm{T}<140 \mathrm{~K})$. The model expresses the total sputtering yield $\mathrm{Y}$ as

$$
Y \quad Y_{n}+Y_{e}
$$

381 with $\mathrm{Y}_{\mathrm{n}}$ being the contribution from collisional sputtering proportional to the nuclear-

382 stopping cross section and $\mathrm{Y}_{\mathrm{e}}$, the contribution from electronic sputtering proportional

383 to the square of the electronic stopping cross section. The former dominates at lower

384 energies, and the latter does so at higher energies. The two components are reflected in the energy spectra of the emitted energetic neutrals, with collisional processes generating more peaked energy spectra (e.g., Figure 4) and electronic sputtering

387 generating an $\mathrm{E}^{-2}$ dependence (e.g., Figure 6). In the collisional domain, it is likely 388 that the emitted energetic neutrals originate from the incident primary ion, whereas in 389 the electronic domain, the emitted energetic neutrals originate from the ice itself. This 390 results in $\mathrm{Y}_{\mathrm{ENA}} \approx \mathrm{Y}_{\mathrm{H}}$ for incident protons at lower energies and $\mathrm{Y}_{\mathrm{ENA}} \approx \mathrm{Y}_{\mathrm{O}}$ at higher 391 incident energies (Figure 8, top panel). $\mathrm{Y}_{\mathrm{ENA}}$ is always smaller than the total sputtering 392 yield because $\mathrm{Y}_{\mathrm{ENA}}$ only includes the energetic neutral particle fraction emitted from 393 the surface with energies above $E_{\min }$. The relative difference between $Y_{\mathrm{ENA}}$ and the 394 total sputtering yield is larger for impinging ion energies below a few $\mathrm{keV}$ because 395 fewer energetic neutrals with energies above $\mathrm{E}_{\min }$ are generated (Wurz and Lammer, 396 2003, Wurz et al. 2007). Additionally, at low impinging ion energy, the obtained $\mathrm{Y}_{\mathrm{ENA}}$

397 is more uncertain due to the assumption of the energetic neutral flux being emitted 398 according to a $\cos ^{4 / 3} \theta$ angular distribution; e.g., for protons impinging on lunar 399 regolith, a tri-modal angular energetic neutral atom flux distribution with an 400 enhancement in both the specular reflection and impinging direction is observed 
401 (Schaufelberger et al., 2012). In our data, $\mathrm{Y}_{\mathrm{ENA}}$ exceeds the model predicted total 402 sputtering yield slightly for impinging hydrogen ions above $20 \mathrm{keV}$, which reflects the 403 uncertainty in the scaling of the model to our application. Impurities in our ice sample $404\left(\mathrm{CO}_{2}\right.$, hydrohalite, air) may also affect the observed energetic neutral particle yield. 405 Impurities can increase the sputtering yield via the formation of more volatile species. 406 The most volatile species in the surface are sputtered most efficiently; however, after 407 sufficient exposure to the impinging ions, the composition of the sputtered material 408 approaches bulk composition (Johnson, 1998). The concentration of impurities in our 409 water ice was a few percent, with the dominant impurity component being the 410 hydrohalite. Although this concentration may affect the observed sputtering yield, a 411 comparative measurement in the same setup with pure water ice or pure hydrohalite 412 would be needed to quantify it. 

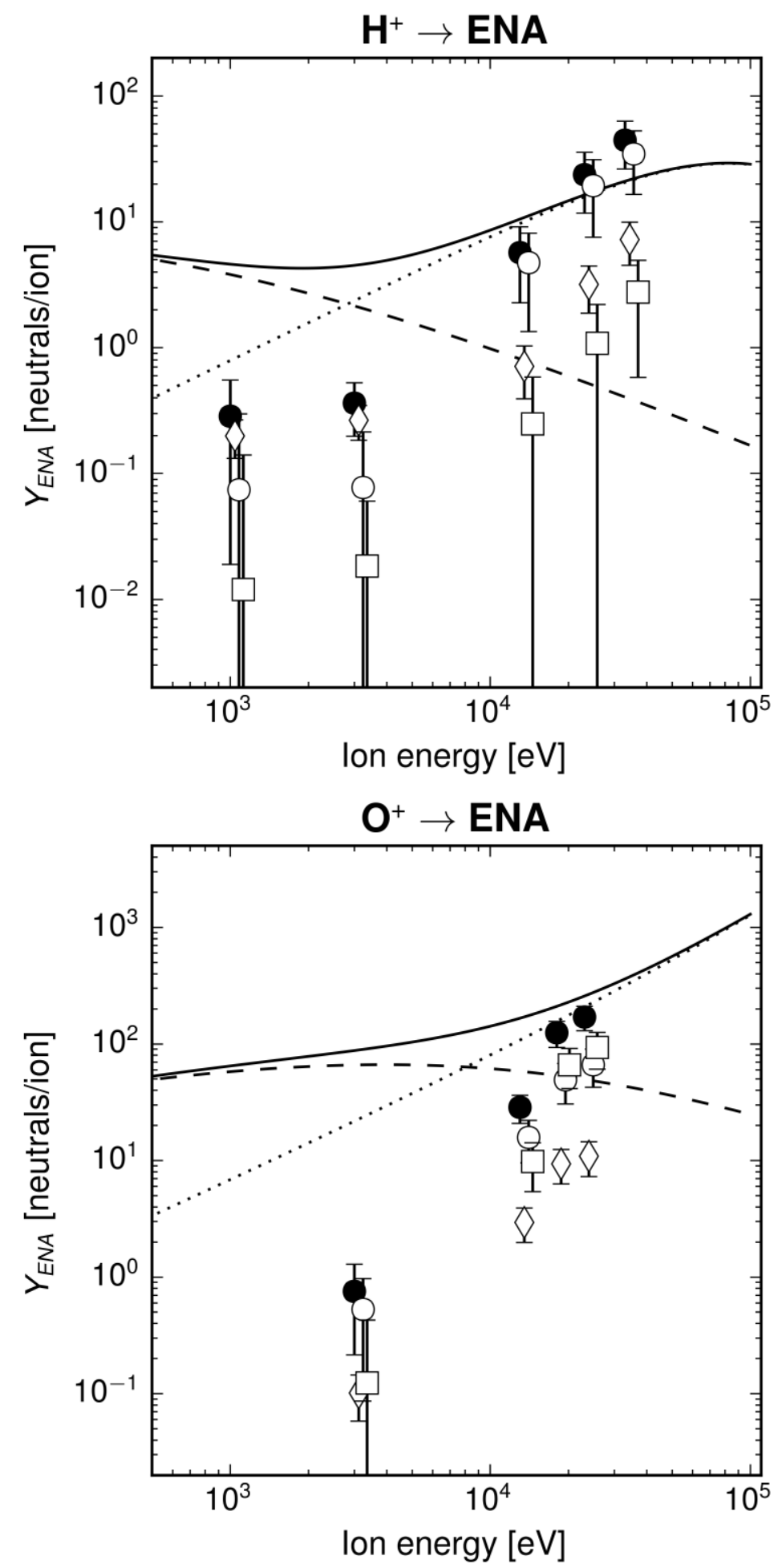

Figure 8: Energetic neutral particle yields, $Y_{E N A}$, for incident protons (top panel) and oxygen ions (bottom panel). Different symbols represent mass groups of emitted neutrals: H (open diamonds), O-group (open circles), Heavy-group (open squares), and their sum (filled circles). The data points for the oxygen-group and the 'heavy' ENAs are slightly shifted to the right on the energy axis for easier reading. For incident oxygen ions of $18 \mathrm{keV}$ and $23 \mathrm{keV}$, the O-group likely consists of $\mathrm{H}_{2} \mathrm{O}$ 
molecules and the corresponding $H$ ENA signal may result from their dissociation in the JNA instrument. The expected total molecular sputtering yield $Y$, modeled after Famà et al. (2008) for an angle of incidence of $60^{\circ}$ (see text), is shown as a solid line for comparison, along with its collisional component $Y_{n}$ (dashed line) and electronic component $Y_{e}$ (dotted line).

\section{Conclusions}

We measured energy spectra of sputtered energetic particles from salty water ice under Ganymede-like conditions under ion bombardment. The energy spectrum of the neutral atom flux emitted from the ice could, in some cases, be fitted with Maxwellian distributions, which is indicative of particle reflection on the surface. For higher incident energies, where electronic sputtering becomes dominant, the energy spectrum changed to a Sigmund-Thompson shape. A very high hydrogen ENA yield of 0.20 for $1 \mathrm{keV}$ incident protons was observed. Hydrogen ENA yields of $\sim 7$ were measured for $33 \mathrm{keV}$ incident protons. Oxygen and other heavier ENAs produced by incident protons generally exhibited a Sigmund-Thompson spectrum, although the intensities were rather low due to mass mismatch between the incident and sputtered particles. The total energetic neutral particle yield for incident protons, with all measured ENA species combined, ranged from 0.28 at $1 \mathrm{keV}$ to $\sim 40$ at $33 \mathrm{keV}$ incident ion energy.

Incident oxygen ions, which favored electronic sputtering in the energy range used, resulted in a yield for oxygen or water ENAs up to 100 for $23 \mathrm{keV}$ incident ion energy. For oxygen ions with an incident energy of $3 \mathrm{keV}$, the observed oxygen ENA yield was 0.5. Additionally, at the same incident energy, a recoil process was observed, producing a distinct hydrogen ENA signal with a yield of 0.10 . Total energetic neutral particle yield for incident oxygen ions ranged from 0.8 at $3 \mathrm{keV}$ to 
$\sim 170$ at $23 \mathrm{keV}$ incident ion energy.

447

448

Acknowledgements

449

P.W. thanks the Swiss National Science foundation for their financial support.

450

451

452

453

References

454

Barabash, S., A. Bhardwaj, M. Wieser, R. Sridharan, T. Kurian, S. Varier, E. Vijayakunar, V. Abhirami, K. V. Raghavendra, S. V. Mohankumar, M. B. Dhanya, S. Thampi, K. Asamura, H. Andersson, Y. Futaana, M. Holmstrom, R. Lundin, J. Svensson, S. Karlsson, R. D. Piazza, P. Wurz, Investigation of the solar windMoon interaction onboard Chandrayaan-1 mission with the SARA experiment, Current Science, 96, 4, 526 (2009)

Barabash, S., P. Wurz, P. Brandt, M. Wieser, M. Holmström, Y. Futaana, G. Stenberg, 461 H. Nilsson, A. Eriksson, M. Tulej, et al. Particle environment package (PEP). European Planetary Science Congress 2013, held 8-13 September in London, UK. Online at: http://meetings. copernicus. org/epsc2013, id. EPSC2013-709, 8:709 (2013).

Baragiola, R. A., R. A. Vidal, W. Svendsen, J. Schou, M. Shi, D. A. Bahr, and C. L. Atteberrry. Sputtering of water ice. Nuclear Instruments and Methods in Physics

467 Research Section B: Beam Interactions with Materials and Atoms, 209(0):294-303

468 (2003). 10.1016/S0168-583X(02)02052-9. URL

469 http://www.sciencedirect.com/science/article/pii/S0168583X02020529 
470 Betz, G. and K. Wien, Energy and angular distributions of sputtered particles, Int. J. Mass Spec. and Ion Proc. 141, (1994)

Cooper, J.F., R. E. Johnson, B. H. Mauk, H. B. Garrett, and N. Gehrels, Energetic ion and electron irradiation of the icy galilean satellites. Icarus, 149(1):133-159, 1 2001. doi: 10.1006/icar.2000.6498.

Famá, M., J. Shi, and R. A. Baragiola. Sputtering of ice by low-energy ions. Surface Science, 602(1):156-161 (2008). URL http://www.sciencedirect.com/science/article/pii/S0039602807009879

Futaana, Y., S. Barabash, M. Wieser, M. Holmstrm, C. Lue, P. Wurz, A. 479 Schaufelberger, A. Bhardwa j, M. B. Dhanya, and K. Asamura. Empirical energy 480 spectra of neutralized solar wind protons from the lunar regolith. J. Geophys. Res., 481 117(E5) (2012). doi:10.1029/2011JE004019.

Futaana, Y., S. Barabash, X.-D. Wang, M. Wieser, G.S. Wieser, P. Wurz, N. Krupp, 483 and P. C:Son Brandt, Low-Energy Energetic Neutral Atom Imaging of Io Plasma and Neutral Tori, Planet. Sp. Sci. 108, (2015), 41_53, doi:10.1016/j.pss.2014.12.022. A. Coates, P. Drossart, L. N. Fletcher, H. Hussmann, R. Jaumann, N. Krupp, J. P. Lebreton, O. Prieto-Ballesteros, P. Tortora, F. Tosi, and T. Van Hoolst, JUpiter ICy moons Explorer (JUICE): An ESA mission to orbit Ganymede and to characterise 490 the Jupiter system, Planet. Space Sci., 78(0), 1-21, doi: 10.1016/j.pss.2012.12.002, 2013 
492 Ip, W.-H., D. J. Williams, R. W. McEntire, and B. Mauk. Energetic ion sputtering 493 effects at Ganymede. Geophysical Research Letters, 24(21):2631-2634 (1997). 494 doi:10.1029/97GL02814.

495

496

497

498

499

500

501

502

503

504

505

506

507

508

509

510

511

512 Küstner, M., Eckstein, W., Dose, V., Roth, J., The influence of surface roughness on

James, P. B., H. H. Kieffer, and D. A. Paige, The seasonal cycle of carbon dioxide on Mars, in Mars, edited by H. H. Kieffer, B. M. Jakosky, C . W. Snyder, and M. S. Matthews, pp.934-968, University of Arizona Press, Tucson, 1992.

Johnson, R. E. Sputtering and Desorption from Icy Surfaces. In B. Schmitt, C. de Bergh, and M. Festou, eds., Solar System Ices, volume 227 of Astrophysics and Space Science Library, page 303 (1998).

Johnson, R. E. Sputtering and Desorption from Icy Surfaces. In B. Schmitt, C. de Bergh, and M. Festou, eds., Solar System Ices, volume 227 of Astrophysics and Space Science Library, page 303 (1998).

Kazama, Y., S. Barabash, M. Wieser, K. Asamura, and P. Wurz, Development of an LENA instrument for planetary missions by numerical simulations, Planet. Space Sci. 55 (2007) 1518-1529.

Khurana, K. K., Euler potential models of Jupiter's magnetospheric field. J. Geophys. Res: Space Physics, 102(A6):11295-11306, 1997. ISSN 2156-2202. doi: 10.1029/97JA00563. (1997)

Khurana, K. K., R. T. Pappalardo, N. Murphy, and T. Denk, The origin of ganymede's polar caps, Icarus, 191(1), 193-202, (2007). 
513 the angular dependence of the sputter yield. Nucl. Instrum. Methods B 145, 320$514 \quad 331,(1998)$.

515 Küstner, M., W. Eckstein, E. Hechtl, and J. Roth. Angular dependence of the 516 sputtering yield of rough beryllium surfaces. Journal of Nuclear Materials, 265(1517 2):22-27, 2 (1999). doi: 10.1016/S0022-3115(98)00648-5.

518 Lancaster, G.M., F. Honda, Y. Fukuda, and J.W. Rabalais, Secondary Ion Mass 519 Spectometry of Molecular Solids: Cluster Formation during Ion Bombardement of 520 Frozen Water, benzene, and Cyclohexane, Jou. Am. Chem. Soc. 101 (1979), 1951$521 \quad 1958$.

Loeffler, M.J., Raut, U., Vidal, R.A., Baragiola, R.A., Carlson, R.W., Synthesis of 523 hydrogen peroxide in water ice by ion irradiation. Icarus 180,530 265-273 (2006).

Loeffler, M. J., C. A. Dukes, and R. A. Baragiola (2009), Irradiation of olivine by 4 keV He+: Simulation of space weathering by the solar wind, J. Geophys. Res., 114, E03003, doi:10.1029/2008JE003249.

McCarthy, C., R. F Cooper, S. H Kirby, K. D Rieck, and L. A Stern. Solidification and 528 microstructures of binary ice-i/hydrate eutectic aggregates. American Mineralogist, 529 92(10):1550-1560, 2007.

McCord, T. B., G. B. Hansen, R. N. Clark, P. D. Martin, C. A. Hibbitts, F. P. Fanale, J. Smythe, and G. E. Danielson. Non-water-ice constituents in the surface material of 533 the icy galilean satellites from the Galileo near-infraredmapping spectrometer 534 investigation. Journal of Geophysical Research: Planets, 103(E4):8603-8626, 

1998. doi: 10.1029/98JE00788.

536 McCord, T. B., RW Carlson, WD Smythe, GB Hansen, RN Clark, CA Hibbitts, FP 537 Fanale, JC Granahan, M Segura, DL Matson, et al. Organics and other molecules $538 \quad$ in the surface of the icy galilean satellites. Science, 278:271-275, 1997.

540 Wind Plasma Instrumentation. Rev. Sci. Instr., 72(2):1354-1360 (2001).

541 Murphy, D. M. and T. Koop, Quarterly Journal of the Royal Meteorological Society $542131(2005)$, no. 608, 1539-1565.

543 Niehus, H., W. Heiland, E. Taglauer, Low-energy ion scattering at surfaces, Surface $544 \quad$ Science Reports 17 (1993) 213-303

545 Schaufelberger, A. et al., Geophys. Res. Lett., 38, L22202, 546 doi:10.1029/2011GL049362, 2011

547 Shi, M., R. A. Baragiola, D. E. Grosjean, R. E. Johnson, S. Jurac, and J. Schou. 548 Sputtering of water ice surfaces and the production of extended neutral 549 atmospheres. Journal of Geophysical Research: Planets, 100(E12):26387-26395 550 (1995). 10.1029/95JE03099. URL http://dx.doi.org/10.1029/95JE03099

551 Showman, A. P., and R. Malhotra. The galilean satellites. Science, 286(5437):77-84, $552 \quad 101999$.

553 Sigmund, P., 1969. Theory of sputtering. I. sputtering yield of amorphous and 554 polycrystalline targets. Phys. Rev. 184, 383-416. 
van Slooten, U., D. Andersson, A. W. Kleyn, and E. A. Gislason. Scaling law for dissociation of fast molecular hydrogen scattered from Ag (111). Chemical Physics Letters, 185(5-6):440-444, 101991.

Vorburger, A., P. Wurz, S. Barabash, M. Wieser, Y. Futaana, C. Lue, M. Holmström, A. Bhardwaj, M. B. Dhanya, and K. Asamura (2013), Energetic neutral atom 560 imaging of the lunar surface, J. Geophys. Res. Space Physics, 118, 3937-3945, doi:10.1002/jgra.50337.

Wieser, M., and P. Wurz. Production of a $10 \mathrm{eV}-1000 \mathrm{eV}$ neutral particle beam using surface neutralization. Meas. Sci. Technol., 16:2511-2516, 2005.

Wieser, M., S. Barabash, Y. Futaana, M. Holmström, A. Bhardwa j, R. Sridharan, M. Dhanya, P. Wurz, A. Schaufelberger, and K. Asamura. Extremely high reflection of solar wind protons as neutral hydrogen atoms from regolith in space. Planetary and Space Science, 57(14-15):2132 - 2134, 2009. doi: 10.1016/j.pss.2009.09.012.

Wieser, M., S. Barabash, Y. Futaana, M. Holmström, A. Bhardwa j, R. Sridharan, M. B. Dhanya, A. Schaufelberger, P. Wurz, and K. Asamura. First observation of a mini-magnetosphere above a lunar magnetic anomaly using energetic neutral atoms. Geophys. Res. Lett., 37(5) (2010). doi:10.1029/2009GL041721.

Wurz, P., and H. Lammer, Monte-Carlo Simulation of Mercury's Exosphere, Icarus, 164(1), (2003), 1-13.

574 Wurz, P., U. Rohner, J.A. Whitby, C. Kolb, H. Lammer, P. Dobnikar, and J.A. Martín575 Fernández, The Lunar Exosphere: The Sputtering Contribution, Icarus 191 (2007), 576 486-496, DOI:10.1016/j.icarus.2007.04.034. 
577 Wurz, P., J.A. Whitby, U. Rohner, J.A. Martín-Fernández, H. Lammer, and C. Kolb, 578 "Self-consistent modelling of Mercury's exosphere by sputtering, micro-meteorite 579 impact and photon-stimulated desorption," Planet. Space Science 58 (2010) 1599$580 \quad 1616$.

581 Wurz, P., U. Rohner, J.A. Whitby, C. Kolb, H. Lammer, P. Dobnikar, and J.A. Martín582 Fernández, "The Lunar Exosphere: The Sputtering Contribution," Icarus 191 583 (2007), 486-496, DOI:10.1016/j.icarus.2007.04.034. 